\title{
Spectral methods for the detection of network community structure: A comparative analysis
}

\author{
Hua-Wei Shen and Xue-Qi Cheng \\ Institute of Computing Technology, Chinese Academy of Sciences, Beijing, China \\ E-mail: shenhuawei@software.ict.ac.cn, cxq@ict.ac.cn
}

\begin{abstract}
Spectral analysis has been successfully applied at the detection of community structure of networks, respectively being based on the adjacency matrix, the standard Laplacian matrix, the normalized Laplacian matrix, the modularity matrix, the correlation matrix and several other variants of these matrices. However, the comparison between these spectral methods is less reported. More importantly, it is still unclear which matrix is more appropriate for the detection of community structure. This paper answers the question through evaluating the effectiveness of these five matrices against the benchmark networks with heterogeneous distributions of node degree and community size. Test results demonstrate that the normalized Laplacian matrix and the correlation matrix significantly outperform the other three matrices at identifying the community structure of networks. This indicates that it is crucial to take into account the heterogeneous distribution of node degree when using spectral analysis for the detection of community structure. In addition, to our surprise, the modularity matrix exhibits very similar performance to the adjacency matrix, which indicates that the modularity matrix does not gain desired benefits from using the configuration model as reference network with the consideration of the node degree heterogeneity.
\end{abstract}

PACS numbers: 89.75.Hc, 89.75.Fb, 05.10.-a 
Spectral methods for the detection of network community structure: A comparative analysis 2

\section{Introduction}

Many complex systems in the real world can be modeled as graphs or networks [1]. The topological characteristics and dynamics on/of networks are critical to understanding the relationship between structure and function of networks, such as the modeling of networks [2], the evolution of networks [3, 4], the resilience of network [5, 6], and capacity of networks [7]. Many real world networks, including social networks [8], information networks [9], and biological networks [10], are found to divide naturally into communities, known as groups of nodes such that the nodes within a group are much more connected to each other than to the rest of the network. Communities are of interest because they often correspond to functional units such as the collections of pages on a single topic on the Web [9] and the pathways for metabolic networks [10].

The identification of community structure has attracted much attention in various scientific fields. Many methods have been proposed and applied successfully to some specific complex networks [11, 12, 13, 14, 15, 16, 17, 18, 19, 20, 21]. For reviews, the reader can refer to [22]. These methods are from different perspectives, such as the centrality measures, link density, percolation theory, and network compression. Besides these methods, the spectral analysis has gained great success at uncovering the community structure, respectively being based on the adjacency matrix [23], the standard Laplacian matrix [24, the normalized Laplacian matrix [25], the modularity matrix [16, 17], the correlation matrix [26] and several other variants of these matrices. However, to our knowledge, the comparison between these different spectral methods is less reported. More importantly, it is still unclear which matrix is more appropriate for the detection of community structure.

In this paper, we conduct a comparative analysis of the aforementioned five matrices on the benchmark networks which have heterogeneous distributions of node degree and community size. The comparison is carried out from two perspectives. The former one focuses on whether the number of intrinsic communities can be exactly identified according to the spectrum of these five matrices. The latter evaluates the effectiveness of these matrices at identifying the intrinsic community structure using their eigenvectors. Test results show that the normalized Laplacian matrix and the correlation matrix significantly outperform the other three matrices. The possible reason is that these two matrices are both normalized using the degree of nodes. Thus we can conclude that it is crucial to take into account the heterogeneous distribution of node degree when using spectral analysis for the detection of community structure. In addition, to our surprise, the modularity matrix exhibits very similar performance to the adjacency matrix, which indicates that the modularity matrix does not gain desired benefits from using the configuration model as reference network with the consideration of the node degree heterogeneity.

Several comparative analyses on the methods for community detection have been conducted [27, 28. Different from them, our comparative analysis focuses on the matrices underlying the spectral methods for community detection rather than 
Spectral methods for the detection of network community structure: A comparative analysis3

comparing the specific implementation of the existing spectral methods with different heuristics to improve the final performance. This comparison is fair and meaningful since the performance of spectral methods heavily relies on the characteristics of the underlying matrices.

\section{The matrices for spectral analysis}

The topology of network is often described in terms of adjacency matrix. Based on the adjacency matrix, several other matrices are formulated to investigate the properties of network, including the standard Laplacian matrix, the normalized Laplacian matrix, the modularity matrix and the correlation matrix. Existing studies indicate that the spectrum of these matrices sheds light on the community structure of network. In the following, we first give the definition of these matrices and briefly introduce the methods to detect the community structure using the spectrum of these matrices.

- Adjacency matrix. The elements $A_{i j}$ of an adjacency matrix $A$ denote the strength of the edge connecting the nodes $i$ and $j$ if such an edge exists, and 0 otherwise. (We restrict our attention in this paper to undirected networks.) In [23, the authors proposed that the spectrum of the adjacency matrix can unravel the number of communities. Specifically, the eigenvalues of the adjacency matrix is ranked in descending order, i.e., $\lambda_{1}^{A} \geq \lambda_{2}^{A} \geq \cdots \geq \lambda_{i}^{A} \geq \cdots \geq \lambda_{n}^{A}$, where $n$ is the number of network nodes. Each two successive eigenvalues form an eigengap, the $i$ th eigengap being between $\lambda_{i}^{A}$ and $\lambda_{i+1}^{A}(1 \leq i \leq n-1)$. The length of the $i$ th eigengap is $\lambda_{i}^{A}-\lambda_{i+1}^{A}$. Then, the number of communities is indicated by the place of the largest eigengap, i.e., $i$ is the number of communities if the largest eigengap is the $i$ th one.

- Standard Laplacian matrix. The standard Laplacian matrix is defined as $L=D-A$, where $D$ is a diagonal matrix with the diagonal element $D_{i i}$ being the degree of the node $i$. As to the standard Laplacian matrix, the Fiedler's vector [29, 30] has been well studied and widely used for two-way network partition. Fiedler's vector is the eigenvector of the standard Laplacian matrix corresponding to the second smallest eigenvalue. More importantly, the standard Laplacian matrix is often used to characterize the synchronization dynamics on networks [24, 31]. In [24], Arenas et al. pointed out that the spectrum of the standard Laplacian matrix reveals the intrinsic topological scales. The eigenvalues are ranked in ascending order and the length of the $i$ th eigengap is defined as $\log \lambda_{i+1}^{L}-\log \lambda_{i}^{L},(2 \leq i \leq n-1) \pm$. Then, $i$ is viewed as the appropriate candidate for the number of intrinsic communities if the $i$ th eigengap is largest.

- Normalized Laplacian matrix. The normalized Laplacian matrix is often defined as $N=I-T$, where $I$ is the identity matrix and $T$ is the transition matrix, which is defined as $T=D^{-1} A$ with the elements $T_{i j}$ being the probability

$\ddagger$ In this paper, we also tested the alternative eigengap defined as $\lambda_{i+1}^{L}-\lambda_{i}^{L},(1 \leq i \leq n-1)$, and the results are similar. 
Spectral methods for the detection of network community structure: A comparative analysis4

that a random walker moves to the node $j$ from the node $i$. The normalized Laplacian matrix is named after the fact that it can be written in the form $N=D^{-1} L$, i.e., normalizing the standard Laplacian matrix with the diagonal matrix $D$ of node degrees. In [32], the authors claimed that the spectrum of the transition matrix $T$ can be used to detect the community structure of networks. Actually, if $\lambda$ is an eigenvalue of the transition matrix, $1-\lambda$ is an eigenvalue of the normalized Laplacian matrix with the same eigenvector. Furthermore, the normalized Laplacian matrix is closely correlated to the diffusion dynamics on networks. Through investigating the diffusion dynamics on networks, Cheng and Shen pointed out [25] that the community structure can be identified through the eigenvalues and eigenvectors of the normalized Laplacian matrix. Specifically, the eigenvalues are ranked in ascending order and the length of the $i$ th eigengap is defined as $\lambda_{i+1}^{N}-\lambda_{i}^{N},(1 \leq i \leq n-1)$. Then, $i$ is viewed as the appropriate candidate for the number of intrinsic communities if the $i$ th eigengap is largest.

- Modularity matrix. The modularity matrix is proposed by Newman as a spectral explanation for the well-known measure, namely modularity, for the quality of network partition [16, 17]. Its elements are defined as

$$
B_{i j}=A_{i j}-\frac{k_{i} k_{j}}{2 m}
$$

where $k_{i}=\sum_{j} A_{i j}$ is the strength of the node $i$ and $2 m=\sum_{i j} A_{i j}=\sum_{i} k_{i}$ is the total strength of all the nodes. In [17], the eigenvectors corresponding to positive eigenvalues are utilized to uncover the community structure of networks. The number of communities can be determined according to the magnitude of the positive eigenvalues. Here, we rank the eigenvalues in descending order and the length of the $i$ th eigengap is defined as $\lambda_{i-1}^{B}-\lambda_{i}^{B},(2 \leq i \leq n)$. Then, $i$ is taken as the number of communities if the $i$ th eigengap has the largest length. Note that, for the purpose of the detection of community structure, only the eigengaps among positive eigenvalues are considered. If all the eigenvalues are negative, no natural community structure exists, i.e., all the nodes belong to a sole community and the community number is 1 . In [26], the modularity matrix is shown to be the biased covariance matrix of network and the spectrum of the covariance matrix is investigated for the detection of the multiscale community structure.

- Correlation matrix. The correlation matrix of network characterizes the correlation coefficients between pairs of nodes. Its element $C_{i j}$ are defined as

$$
C_{i j}=\frac{B_{i j}}{\sqrt{k_{i}-k_{i}^{2} / 2 m} \sqrt{k_{j}-k_{j}^{2} / 2 m}} .
$$

In [26], the correlation matrix is used to uncover the multiscale community structure of networks. Specifically, the eigenvalues are ranked in descending order and the length of the $i$ th eigengap is defined as $\lambda_{i-1}^{C}-\lambda_{i}^{C},(2 \leq i \leq n)$. The same to the modularity matrix, only the eigengaps among positive eigenvalues are considered. Then, $i$ is taken as the number of communities if the $i$ th eigengap has the largest 
Spectral methods for the detection of network community structure: A comparative analysis5

length. A similar matrix is called the symmetric normalized Laplacian matrix, whose element at the place $(i, j)$ is defined as $\delta_{i j}-A_{i j} / \sqrt{k_{i}} \sqrt{k_{j}}$, where $\delta_{i j}$ is 1 when $i=j$ and 0 otherwise. This matrix is often used in spectral clustering algorithms together with the two aforementioned Laplacian matrices [33].

In summary, the number of communities can be determined according to the eigengaps of the aforementioned five matrices. Actually, the community structure can be further identified using the eigenvectors of these matrices. Generally speaking, only several eigenvectors are utilized to project each node into a low-dimensional node vectors, and then the community structure is identified through clustering the node vectors using, for example, the $k$-means clustering method. Specifically, the selected eigenvectors correspond to the largest $n_{c}$ eigenvalues for the adjacency matrix, the smallest $n_{c}$ eigenvalues for the standard Laplacian matrix and the normalized Laplacian matrix, the largest $n_{c}-1$ eigenvalues for the modularity matrix and the correlation matrix. Here, $n_{c}$ is the number of communities. These selected eigenvectors are stacked as columns of a matrix and the transpose of the $i$ th row of this matrix is taken as the projected node vector corresponding to the node $i$. The community structure is then detected through clustering the projected node vectors.

Before proceeding, we first clarify why we choose the general method for community detection using the $k$-means clustering method. On one hand, this paper only considers the performance of the aforementioned five matrices rather than the specific implementation of spectral methods. Thus, it is fair and reasonable to choose the general method, which determines the community number according to the spectrum of matrices and identifies the community structure using the eigenvectors of the matrices. On the other hand, the $k$-means clustering method is the common practice for the spectral clustering [33. Furthermore, the $k$-means clustering method is facilitated by the projected node vector subspace spanned by the top eigenvectors of matrices [34]. Thus, using the $k$-means clustering on the node vectors provides a competitive candidate among all the spectral methods for the detection of community structure.

Now, as an example, we illustrate the spectral methods through application on the Zachary's karate club network, which has been widely used to evaluate the community detection methods. This network characterizes the social interactions between the individuals in a karate club at an American university. A dispute arose between the club's administrator and its principal karate teacher, and as a result the club eventually split into two smaller clubs, centered around the administrator and the teacher respectively. The network and its fission is depicted in figure 1. The administrator and the teacher are represented by nodes 1 and 33 respectively. Figure 2 shows the spectrum of the aforementioned five matrices associated with the Zachary's karate club network. The largest eigengap of the adjacency matrix, the standard Laplacian matrix and the modularity matrix indicate that the optimal number of community is 2 . The corresponding community structure is consistent with the real split of the network. However, as indicated by the largest eigengap of the normalized Laplacian matrix and the correlation matrix, 4 is the optimal number of communities. The corresponding four 


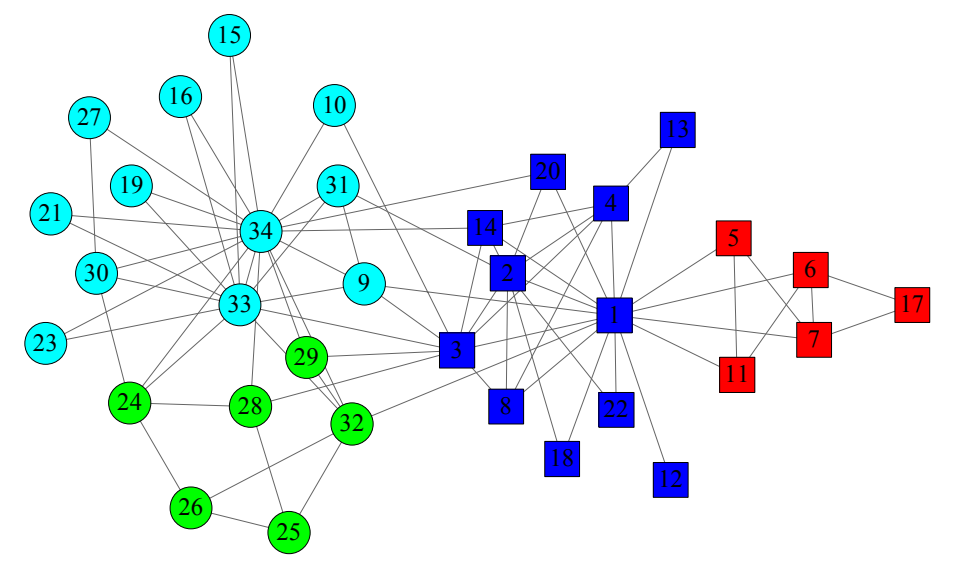

Figure 1. The network of the karate club studied by Zachary [35]. The real social fission of this network is represented by two different shapes, circle and square. Another meaningful partition is often found as the results of many community detection method. The corresponding four communities are differentiated by colors.
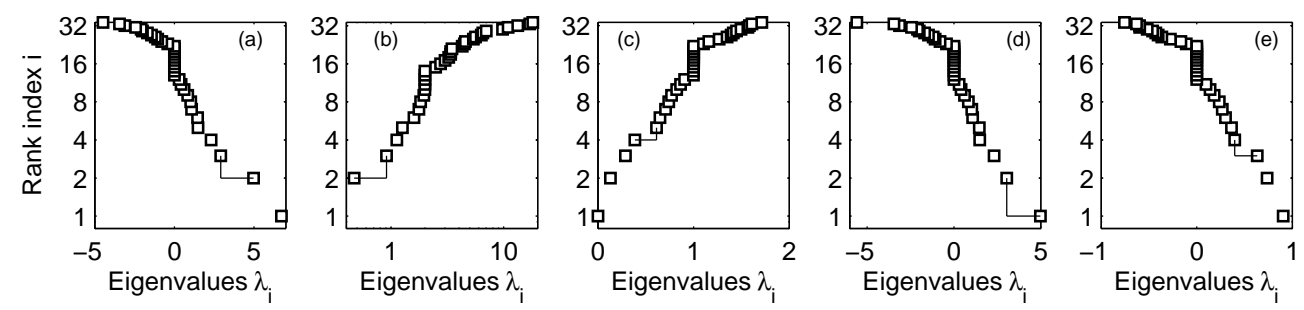

Figure 2. The spectrum of the five considered matrices associated with the Zachary's karate club network, respectively being (a) the adjacency matrix, (b) the standard Laplacian matrix, (c) the normalized Laplacian matrix, (d) the modularity matrix and (e) the correlation matrix. For each matrix, the largest eigengap is marked with an elbow line.

communities are shown in figure 1 differentiated with colors, which is the results of many existing methods for community detection including the modularity maximization.

As illustrated by the previous example, the five matrices give rise to two different resulting partitions as the community structure of the network. Actually these two partitions correspond to two different topological scales of the network. The multiple scale of topological description is a common phenomenon in real-world networks [20, 26, 36, 37, 38, 39, 40]. Actually, the multiscale community structure can be revealed through considering more eigengaps besides the largest one among the eigenvalues of the aforementioned five matrices. As an example, we illustrate the detection of the multiscale community structure of the H13-4 network, which is constructed according to [24]. This network has two predefined hierarchical levels. The first hierarchical level consists of 4 groups of 64 nodes and the second hierarchical level consists of 16 groups of 16 nodes. On average, each node has 13 edges connecting to the nodes in the same group at the second hierarchical level and has 4 edges connecting to the nodes in the same group at the first hierarchical level. This explains the name 
Spectral methods for the detection of network community structure: A comparative analysis7
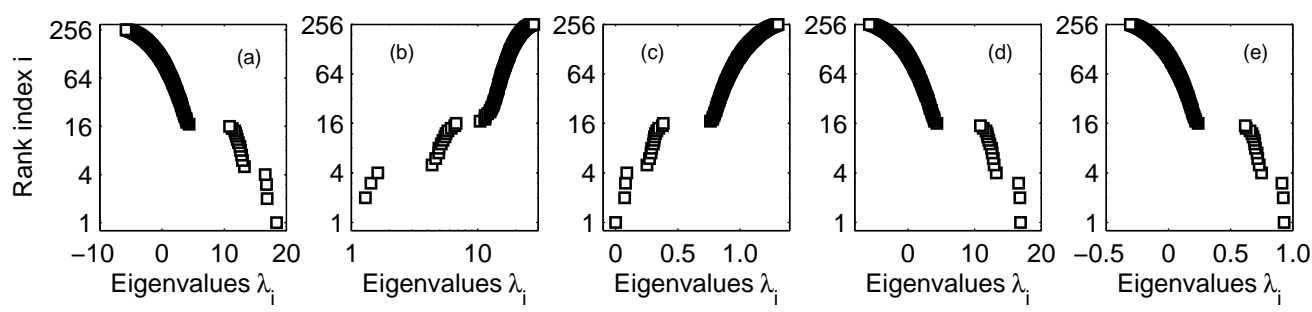

Figure 3. The spectrum of the five matrices associated with the H13-4 network, respectively being (a) the adjacency matrix, (b) the standard Laplacian matrix, (c) the normalized Laplacian matrix, (d) the modularity matrix and (e) the correlation matrix.
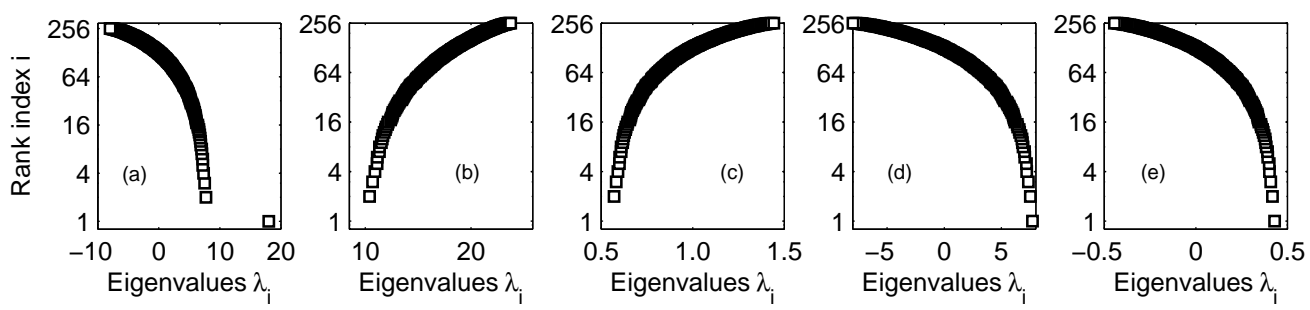

Figure 4. The spectrum of the five matrices associated with the randomized H134 network, respectively being (a) the adjacency matrix, (b) the standard Laplacian matrix, (c) the normalized Laplacian matrix, (d) the modularity matrix and (e) the correlation matrix.

of such kind of networks. In addition, the average degree of each node is 18. According to the construction rules of the H13-4 network, the two hierarchical levels constitute the different topological descriptions of the community structure of the H13-4 network at different scales. As shown in figure 3, the community numbers associated with the two predefined topological scales are clearly revealed by the top two largest eigengaps occurring in the spectrum of the five matrices. The resulting communities are exactly the predefined node groups under the two hierarchical levels. However, according to the length of eigengap, the standard Laplacian matrix seems to prefer the first hierarchical level while the other four matrices tend to reveal the second hierarchical level.

Furthermore, we apply all these matrices to the random network. For comparison, we construct the random network through shuffling the edges of the H13-4 network. Figure 4 shows the spectrum of the five matrices associated with the randomized H134 network. The spectrum of these matrices indicate that the number of communities is 1 or 256 , i.e., all the nodes belong to the same community or each node forms a community. This findings are reasonable since it is commonly believed that randomized networks have no community structure.

The previous examples show that the aforementioned five matrices are both effective at revealing the community structure of network. Note that, as to the example H134 network, the nodes have approximately the same degree and the communities at a specific scale are of the same size. However, the real world networks usually have 
heterogenous distributions of node degree and community size. Thus it will be more convincing to test these matrices on networks with heterogenous distributions of node degree and community size. Before we give such a test in the subsequent section, using a schematic network, we first illustrate the difference between the effectiveness of these matrices. The schematic network is often called the clique circle network as depicted in figure 5. Generally speaking, the intrinsic community structure corresponds to the partition where each clique is taken as a community, which is the sole intrinsic scale existing in this network. As shown in figure 6, the sole topological scale is exactly revealed by the spectrum of the standard Laplacian matrix, the normalized Laplacian matrix and the correlation matrix. However, two scales are observed when we investigate the community structure of this network using the spectrum of the adjacency matrix and the modularity matrix. One scale corresponds to the intrinsic scale of the network, and the other corresponds to the partition dividing the network nodes into 5 groups, which is not desired. In [36], Fortunato et al pointed out the resolution limit problem of the modularity through investigating the modularity maximization on such a clique circle network with each clique having the same size. However, as shown in figure 7, when all the cliques have the same size (i.e., the homogeneous node degree), the intrinsic community structure can be exactly revealed by all the five matrices, including the modularity matrix. This indicates that the resolution limit problem of the modularity is not the same to the problem studied in this paper. Specifically, the resolution limit problem means that there exists an intrinsic scale beyond which the smaller communities cannot be detected through maximizing the modularity. As

to the heterogeneity problem of the modularity matrix considered in this paper, we focus on whether the modularity matrix can reveal the natural community structure, which can be detected using the spectral clustering method instead of the modularity maximization. In sum, the resolution limit problem talks about the maximization of modularity while the heterogeneity problem takes root in the modularity matrix. Thus we claim that it is crucial to deal with the heterogeneous degree when using the spectral methods for community detection.

\section{Tests on benchmark networks}

In this section, we show the effectiveness of the aforementioned five matrices at identifying the community structure on benchmark networks. We utilize the benchmark proposed by Lancichinetti et al. [41]. This benchmark provides networks with heterogeneous distributions of node degree and community size. Thus it poses a much more severe test to community detection algorithms than Newman's standard benchmark [11]. Many parameters are used to control the generated networks in this benchmark: the number of nodes $N$, the average node degree $\langle k\rangle$, the maximum node degree $\max \_k$, the mixing ratio $\mu$, the exponent $\gamma$ of the power law distribution of node degree, the exponent $\beta$ of the power law distribution of community size, the minimum community size min_c, and the maximum community size max_c. In our tests, we 


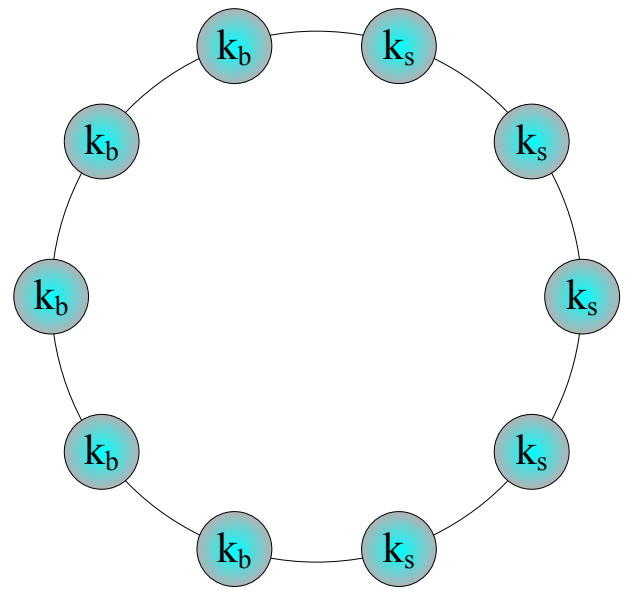

Figure 5. The clique circle network as a schematic example. Each circle corresponds to a clique, whose size is marked by its label $k_{s}$ or $k_{b}$.
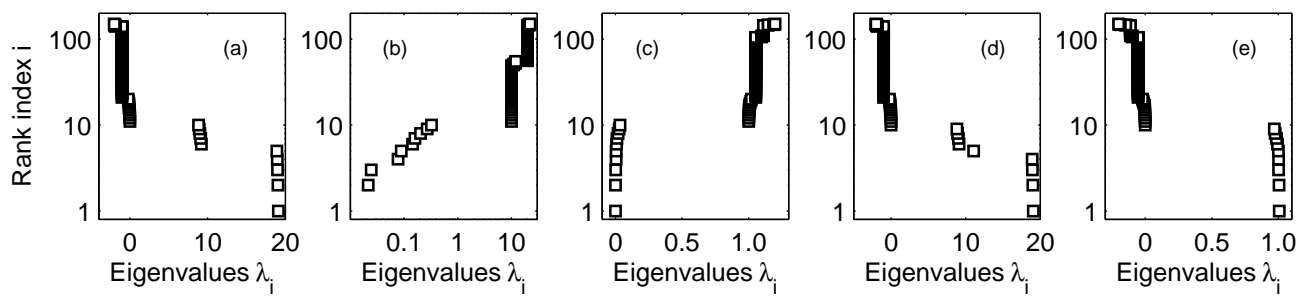

Figure 6. The spectrum of the five matrices on the clique circle network with the clique size being $k_{s}=10$ and $k_{b}=20$. These matrices are respectively (a) the adjacency matrix, (b) the standard Laplacian matrix, (c) the normalized Laplacian matrix, (d) the modularity matrix and (e) the correlation matrix.
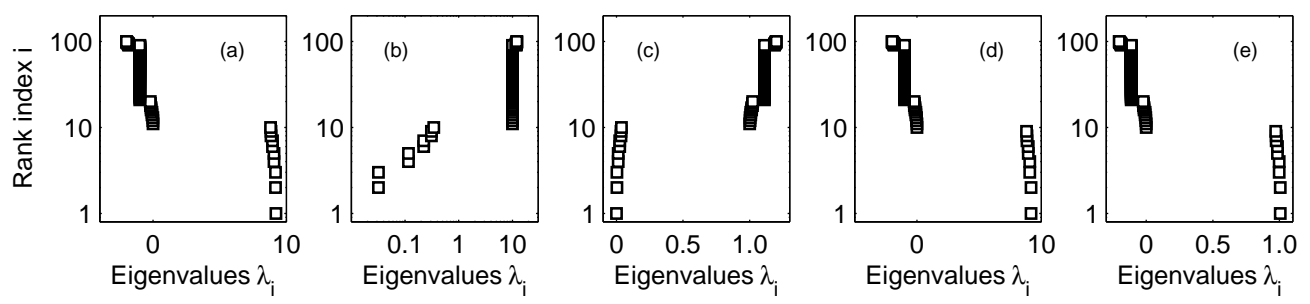

Figure 7. The spectrum of the five matrices on the clique circle network with the clique size being $k_{s}=k_{b}=10$. These matrices are respectively (a) the adjacency matrix, (b) the standard Laplacian matrix, (c) the normalized Laplacian matrix, (d) the modularity matrix and (e) the correlation matrix.

use the default parameter configuration where $N=1000,\langle k\rangle=15, \max \_k=50$, $\min \_c=20$, and $\max \_c=50$. To test the influence from the distribution of node degree and community size, we adopt four parameter configurations for $\gamma$ and $\beta$, respectively being $(\gamma, \beta)=(2,1),(\gamma, \beta)=(2,2),(\gamma, \beta)=(3,1)$ and $(\gamma, \beta)=(3,2)$. Finally, by tuning the parameter $\mu$, we test the effectiveness of the five matrices on the networks with different fuzziness of community structure. The larger the mixing ratio parameter 
Spectral methods for the detection of network community structure: A comparative analysis10
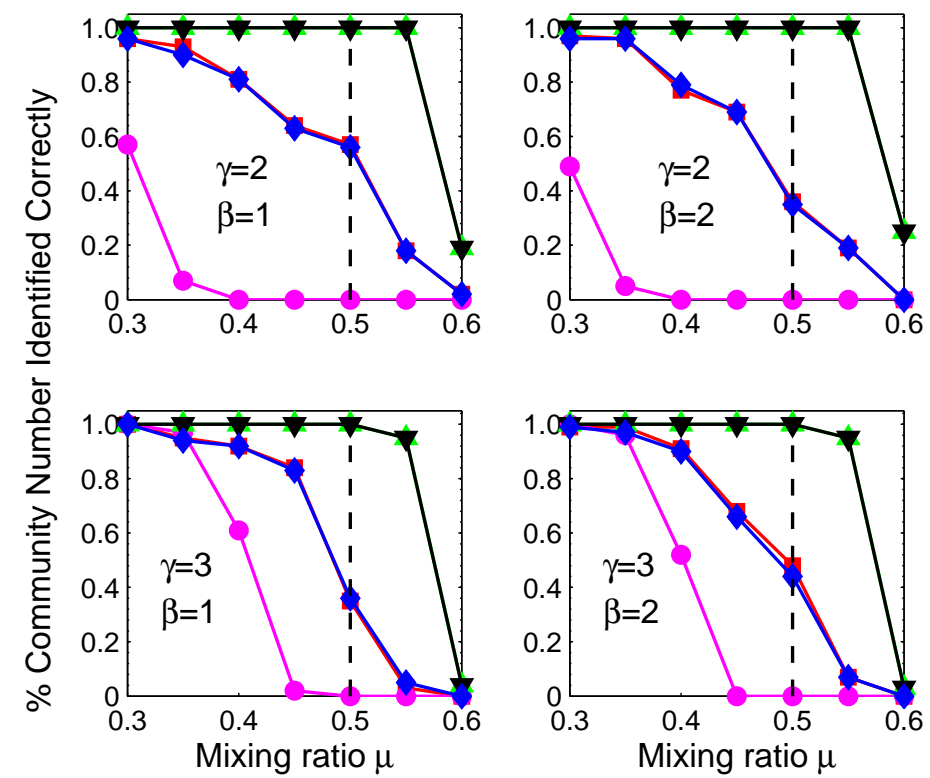

Figure 8. The effectiveness of the five spectral methods at identifying exactly the number of intrinsic communities on benchmark networks with different parameter configurations. For each parameter configuration, 100 generated networks are used. The corresponding matrices are respectively the adjacency matrix $(\square)$, the standard Laplacian matrix $(\bigcirc)$, the normalized Laplacian matrix $(\triangle)$, the modularity matrix $(\diamond)$ and the correlation matrix $(\nabla)$.

$\mu$, the fuzzier the community structure of the generated network.

The first test focuses on whether the number of communities can be correctly identified. Note that each benchmark network has only one significant topological scale according to the construction rules. Thus we only consider whether such a scale can be revealed by the largest eigengap in the spectrum of the five matrices. For each given mixing ratio $\mu, 100$ benchmark networks are generated. For each network, we use the spectrum of the aforementioned five matrices to identify the number of communities. The performance of each method is characterized by the fraction of benchmark networks whose community number is correctly identified. As shown in figure 8 , the best results are obtained by the methods based on the normalized Laplacian matrix and the correlation matrix, which actually give the identical results for all the four used parameter configurations. When the mixing ratio $\mu$ is smaller than 0.5 , i.e., the communities are defined in the strong sense [13], the number of communities can be accurately identified by investigating the spectrum of the normalized Laplacian matrix or the correlation matrix. Even when $\mu$ is larger than 0.5 (e.g., 0.55), these two matrices still give rather good results. The adjacency matrix and the modularity matrix exhibit rather similar effectiveness, obtaining very good results when the community structure is evident and deteriorating when the community becomes fuzzier with the increase of the mixing ratio $\mu$. Compared with the other four matrices, the standard Laplacian 

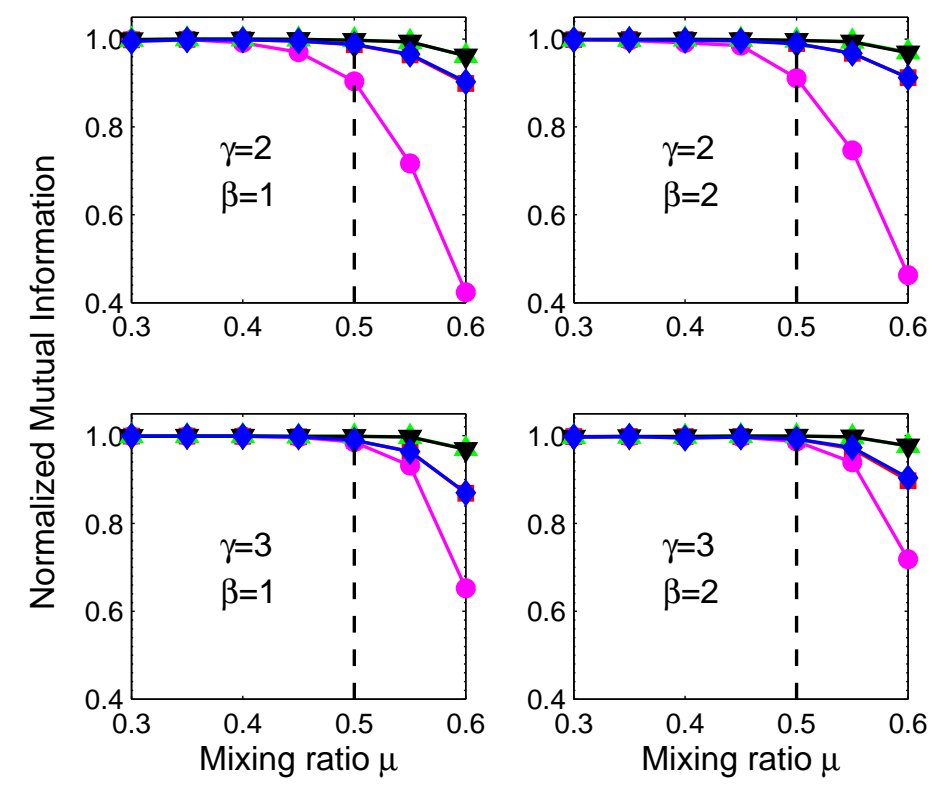

Figure 9. The effectiveness of the five spectral methods at identifying intrinsic community structure on benchmark networks with different configuration parameters. Each point corresponds to an average over 100 network realizations for each parameter configuration. The corresponding matrices are respectively the adjacency matrix $(\square)$, the standard Laplacian matrix $(\bigcirc)$, the normalized Laplacian matrix $(\triangle)$, the modularity matrix $(\diamond)$ and the correlation matrix $(\nabla)$.

matrix gives the worst results, failing to identify the correct number of communities even when the community structure is quite evident. In addition, the exponent $\gamma$ of the power law distribution of node degree affects the effectiveness of the matrices except the normalized Laplacian matrix and the correlation matrix. The possible reason is that these two matrices take into account the distribution of node degree through the normalization operation in their definition. Finally, as shown in figure 8 , it seems that all these five matrices are not very sensitive to the exponent $\beta$ of the power law distribution of community size.

The second test turns to the performance of the eigenvectors of the five tested matrices. Given the number of communities, we investigate whether the predefined community structure can be identified using the eigenvectors of the five tested matrices. The corresponding community detection methods cluster the projected node vectors using the $k$-means clustering method. Each method produces a network partition to represent the community structure. To compare the partition found by these methods with the answer network partition, we adopt the normalized mutual information (NMI) [27] to reflect the effectiveness of each method. The larger the NMI, the more effective the tested method. As shown in figure 9, the same to the first test, the normalized Laplacian matrix and the correlation matrix give the best and almost identical results. The adjacency matrix and the modularity matrix also exhibit the 
Spectral methods for the detection of network community structure: A comparative analysis 12

similar performance, being a little worse than the normalized Laplacian matrix and the correlation matrix. As to the standard Laplacian matrix with the worst performance, the NMI even reaches 0.4 when the mixing ratio $\mu$ is up to 0.6 with $\gamma=2$. Furthermore, the heterogeneous distribution of the node degree affects the NMI of the spectral methods based on the adjacency matrix, the modularity matrix and especially the standard Laplacian matrix.

In summary, the normalized Laplacian matrix and the correlation matrix outperforms the other three matrices both at identifying the number of communities according to the spectrum and identifying the community structure using the top eigenvectors. This indicates that it is crucial to take into account the heterogeneous distribution of node degree when using spectral analysis for the detection of community structure. In addition, although the modularity considers the heterogeneity through introducing the null-model reference network (i.e., the configuration model), as shown in [26], this operation is in fact a kind of translation transformation and thus cannot alleviate the influence on the detection of community structure from the heterogeneous distribution of node degree. This phenomenon can be seen from the experimental results on the Lancichinetti's benchmark networks, i.e., the modularity matrix obtains very similar results to the adjacency matrix.

\section{Conclusions}

We have carried out a comparative analysis on the spectral methods for the detection of network community structure through evaluating the performance of five widely used matrices on the benchmark networks with heterogeneous distribution of node degree and community size. These five matrices are respectively the adjacency matrix, the standard Laplacian matrix, the normalized Laplacian matrix, the modularity matrix and the correlation matrix. Test results demonstrate that the normalized Laplacian matrix and the correlation matrix significantly outperform the other three matrices at identifying the community structure of networks. This indicates that the heterogeneity of node degree is a crucial ingredient for the detection of community structure using spectral methods and the matrices that do not properly account for it are doomed to fail or to give not very accurate results. In addition, to our surprise, the modularity matrix does not gain desired benefits from using the configuration model as reference network with the consideration of the node degree heterogeneity.

\section{Acknowledgments}

This work was funded by the National Natural Science Foundation of China under grants Nos 60873245 and 60933005, and the National Basic Research Program of China (973) under grant No 2007CB310805. The authors gratefully acknowledge S Fortunato and A Lancichinetti for providing the test benchmark. The authors thank the anonymous reviewers for valuable comments on this paper. The authors also thank J M Huang, P 
Spectral methods for the detection of network community structure: A comparative analysis13

Du, X F Zhu, and P Cao for useful discussions and suggestions.

\section{References}

[1] Newman M E J, 2003 SIAM Rev. 45167

[2] Cheng X Q, Ren F X, Zhou S, and Hu M B, 2009 New J. Phys. 11033019

[3] Zhang G Q, Zhang G Q, Yang Q F, Cheng S Q, and Zhou T, 2008 New J. Phys. 10123027

[4] Zhang G Q, Quoitin B and Zhou S, 2010 Comput. Commun. at press, doi: 10.1016/j.comcom.2010.06.004

[5] Albert R, Jeong H, and Barabási A.-L., 1999 Nature 406378

[6] Cheng X Q, Ren F X, Shen H W, Zhang Z K and Zhou T, 2010 J. Stat. Mech. P10011

[7] Zhang G Q, Wang D, and Li G J, 2007 Phys. Rev. E 76017101

[8] Girvan M and Newman M E J, 2002 Proc. Natl. Acad. Sci. 997821

[9] Flake G W, Lawrence S, Giles C L, and Coetzee F M, 2002 Computer 3566

[10] Guimerà R, and Amaral L A N, 2005 Nature 433895

[11] Newman M E J and Girvan M, 2004 Phys. Rev. E 69026113

[12] Clauset A, Newman M E J, and Moore C, 2004 Phys. Rev. E 70066111

[13] Radicchi F, Castellano C, Cecconi F, Loreto V, and Parisi D, 2004 Proc. Natl. Acad. Sci. 1012658

[14] Palla G, Derényi I, Farkas I, and Vicsek T, 2005 Nature 435814

[15] Duch J and Arenas A, 2005 Phys. Rev. E 72027104

[16] Newman M E J, 2006 Proc. Natl. Acad. Sci. 1038577

[17] Newman M E J, 2006 Phys. Rev. E 74036104

[18] Rosvall M and Bergstrom C T, 2007 Proc. Natl. Acad. Sci. 1047327

[19] Bagrow J P, 2008 J. Stat. Mech. P05001

[20] Shen H W, Cheng X Q, Cai K, and Hu M B, 2009 Physica A 3881706

[21] Shen H W, Cheng X Q, and Guo J F, 2009 J. Stat. Mech. P07042

[22] Fortunato S, 2010 Phys. Rep. 48675

[23] Chauhan S, Girvan M, and Ott E, 2009 Phys. Rev. E 80056114

[24] Arenas A, Díaz-Guilera A, and Pérez-Vicente C J, 2006 Phys. Rev. Lett. 96114102

[25] Cheng X Q and Shen H W, 2010 J. Stat. Mech. P04024

[26] Shen H W, Cheng X Q, and Fang B X, 2010 Phys. Rev. E 82016114

[27] Danon L, Díaz-Guilera A, Duch J, and Arenas A, 2005 J. Stat. Mech. P09008

[28] Lancichinetti A and Fortunato S, 2009 Phys. Rev. E 80056117

[29] Fiedler M, 1973 Czechoslovak Mathematical Journal 23298

[30] Fiedler M, 1975 Czechoslovak Mathematical Journal 25619

[31] Yan G, Chen G R, Lü J H, and Fu Z Q, 2009 Phys. Rev. E 80056116

[32] Capocci C, Servedio V D P, Caldarelli G, and Colaiori F, 2005 Physica A 352669

[33] von Luxburg U, 2008 Stat. Comput. 17395

[34] Ding C and He X, 2004 Proc. of 21st Int'l Conf. Machine Learning (ICML2004, Canada), pp 225-232

[35] Zachary W W, 1977 J. Anthropol. Res. 33452

[36] Fortunato S, and Barthélemy M, 2007 Proc. Natl. Acad. Sci. 10436

[37] Arenas A, Fernández A, and Gómez S, 2008 New J. Phys. 10053039

[38] Lambiotte R, Delvenne J C, and Barahona M, 2008 arXiv:0812.1770

[39] Ronhovde P and Nussinov Z, 2009 Phys. Rev. E 80016109

[40] Ahn Y Y, Bagrow J P, and Lehmann S, 2010 Nature 466761

[41] Lancichinetti A, Fortunato S, and Radicchi F, 2008 Phys. Rev. E 78046110 